\title{
THE ROYAL INFIRMARY OF EDINBURGH
}

\section{THE NEW DERMATOLOGICAL AND SPECIAL PAVILION}

THERE are several new buildings in course of erection at the west side of the infirmary grounds, and the first of these, devoted to the treatment of dermatological cases and venereal diseases, is now in use. The formal opening was performed by Sir Norman Walker on June 5th. Exclusive of fittings and furnishings, the new pavilion has been erected at a cost of $£ 40,000$.

The nature and dimensions of this site did not permit of a free plan, but all the large wards are placed clear of the adjoining buildings and the smaller wards are facing south.

The general design is on modern lines, and the vertical treatment gives relief to the short space from the adjoining buildings. The east section has step gables and slate roof to harmonise with the architecture of the old buildings adjoining. The accompanying photograph indicates how successfully the architect has succeeded in combining the outline of a modern building with the existing architectural features of the surrounding infirmary wards and departments. The roof space provides accommodation for the water tank and ventilation fans. The remainder of the roof is flat in order that the light may not be unduly cut off from the Eye Pavilion.

Ferro-concrete was used throughout for the foundations and the basement walls, the remainder of the structure being of steel frame construction encased in concrete, and ferro-concrete floors, in accordance with the most modern practice for fire-resisting buildings. The outer walls are built entirely of cast or synthetic stone with inner lining of brickwork.

There are five floors with a basement in the building, arranged as follows :-

Ground Floor.-V.D. Male Out-patients.

First Floor.-V.D. Male In-patients and Lecture Theatre. 


\section{BRITISH JOURNAL OF VENEREAL DISEASES}

Second Floor.-V.D. Female In-patients' and Outpatients' Department.

Third Floor.-Skin Female In-patients' and Children's Wards.

Fourth Floor.-Skin Male In-patient and General Outpatients' Department.

A separate entrance is given to the V.D. male outpatient section at the north-east corner adjoining the

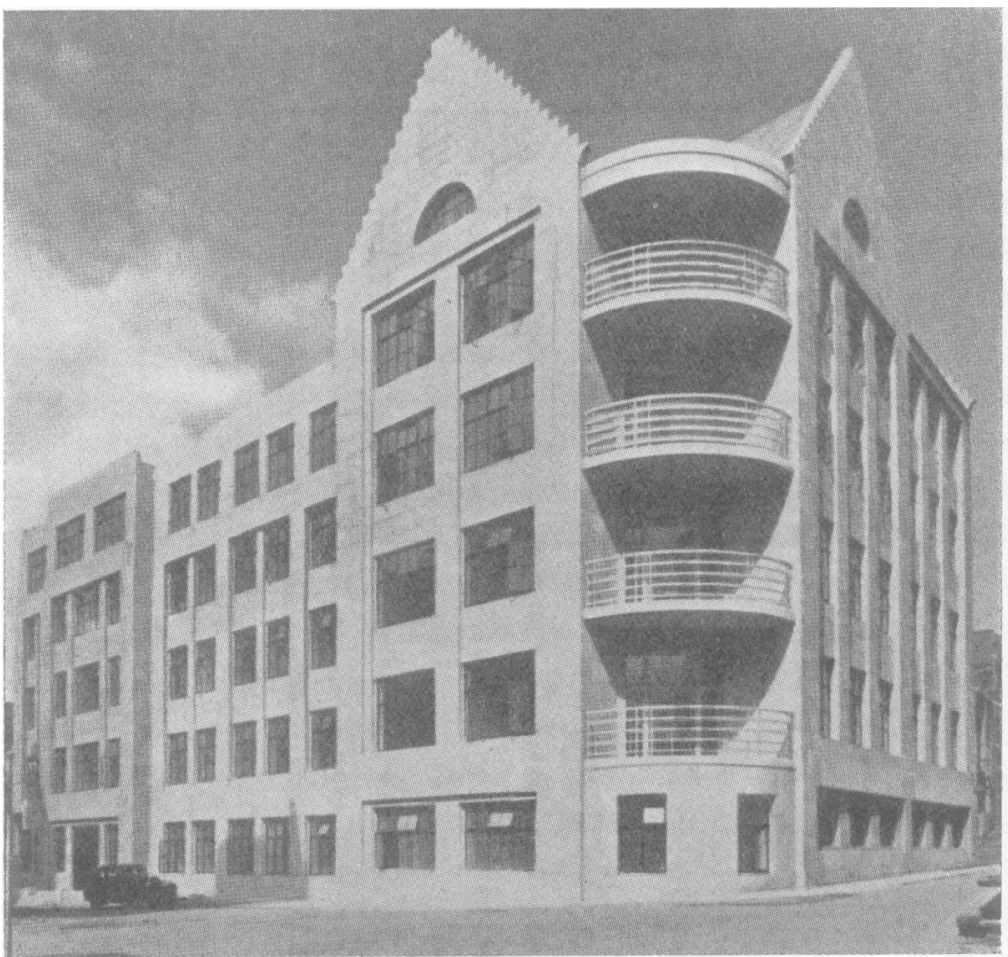

The New Dermatological and Special Pavilion, Royal Infirmary of Edinburgh, 1936. ("From the Edinburgh Medical Journal, Vol. xliii., No. 7.")

west roadway. The entrance hall has a service window to the record office and opens into a large waiting hall, from which doors lead to the treatment rooms and irrigation station. A call and signal light system is arranged from the treatment rooms to the waiting hall and office. In the irrigation station stalls of aluminium and glass have been erected with special fireclay troughs in white enamel and constant flushing system; also a sterile hot water supply at constant temperature is laid on.

A main entrance to all the upper floors is situated at 
THE ROYAL INFIRMARY OF EDINBURGH

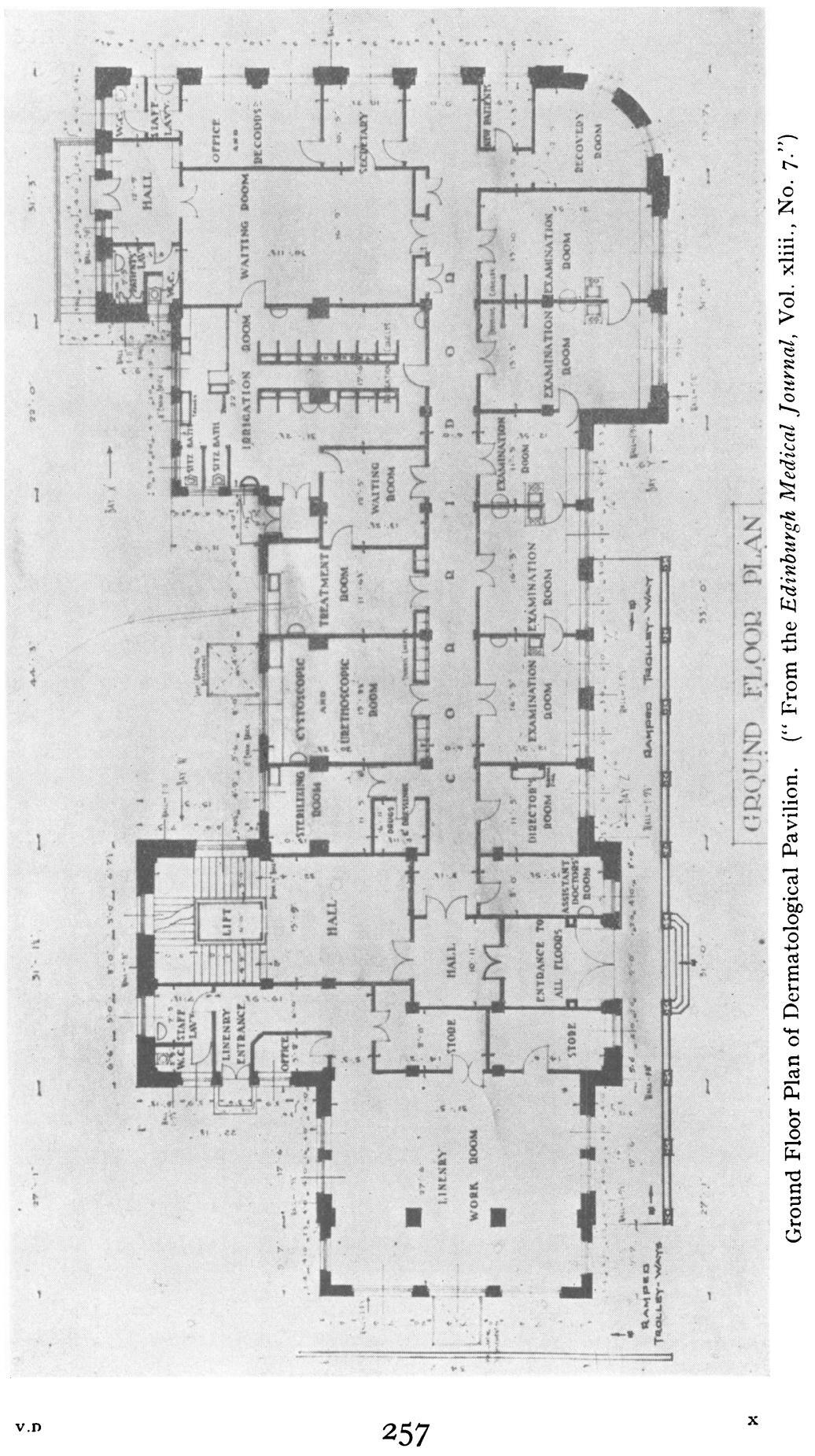




\section{BRITISH JOURNAL OF VENEREAL DISEASES}

the south side, with outer and inner halls leading to the main stairway and lift. A Waygood-Otis lift of the latest type, with steel car and true-level device for landings, is installed, and the enclosure is of aluminium and glass

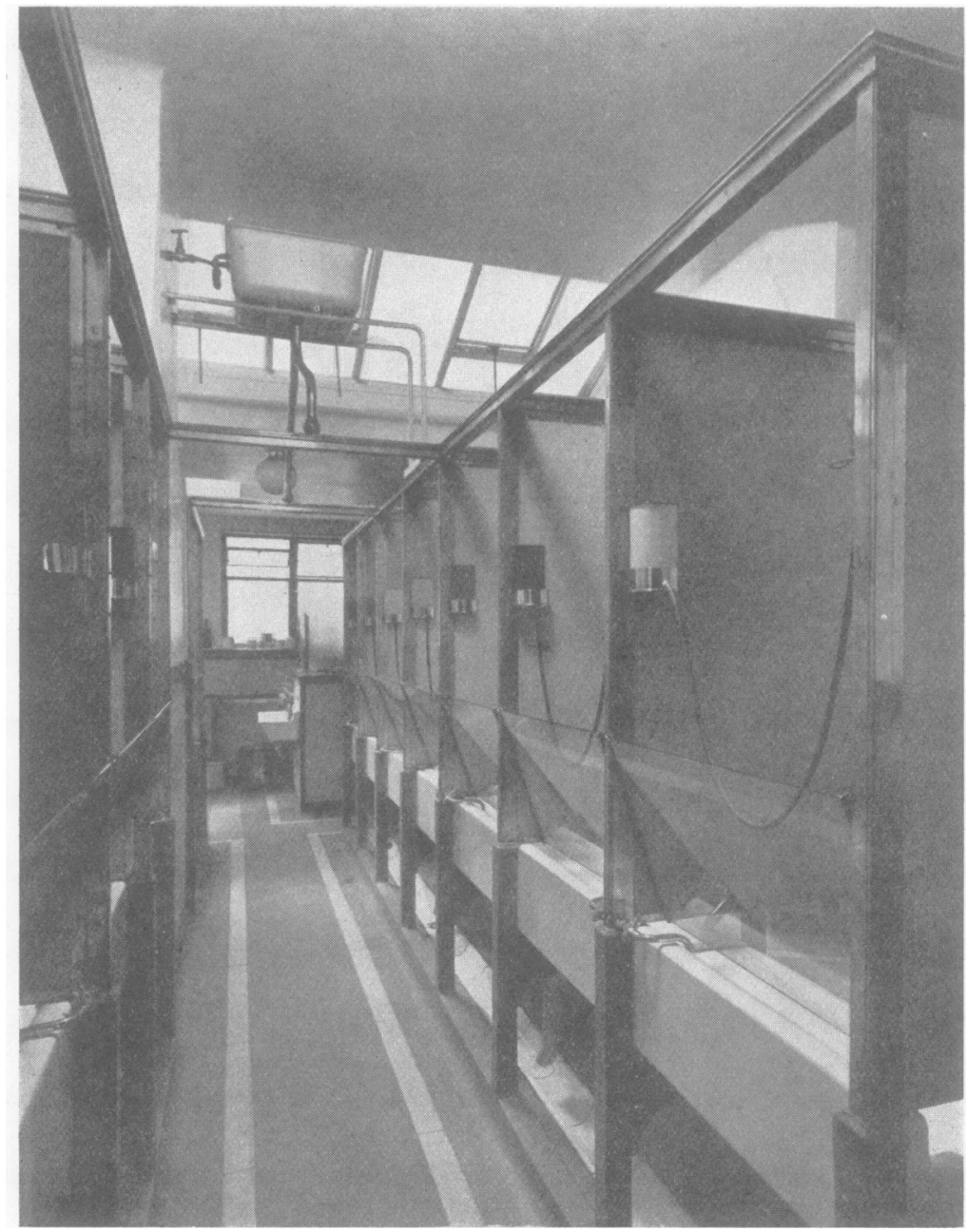

View of Irrigation Room.

throughout, the whole giving a clean treatment with a plentiful supply of light.

The large wards on each floor are at the east side of the building, and each ward has a balcony at the southeast corner. The wards in the V.D. section contain fourteen beds each, and, in the skin section, thirteen 258 


\section{THE ROYAL INFIRMARY OF EDINBURGH}

beds each. Special baths have been fitted in the skin wards.

On the first floor, in addition to the V.D. male ward unit, a lecture room for the joint use of both departments is provided.

The V.D. female ward is on the second floor, and the west section is separated therefrom and arranged for the female out-patients. In this section there is a suitable waiting room, record office, four treatment rooms, etc.

Wards for skin female patients are arranged on the third floor.

On the fourth (top) floor this dermatological ward is

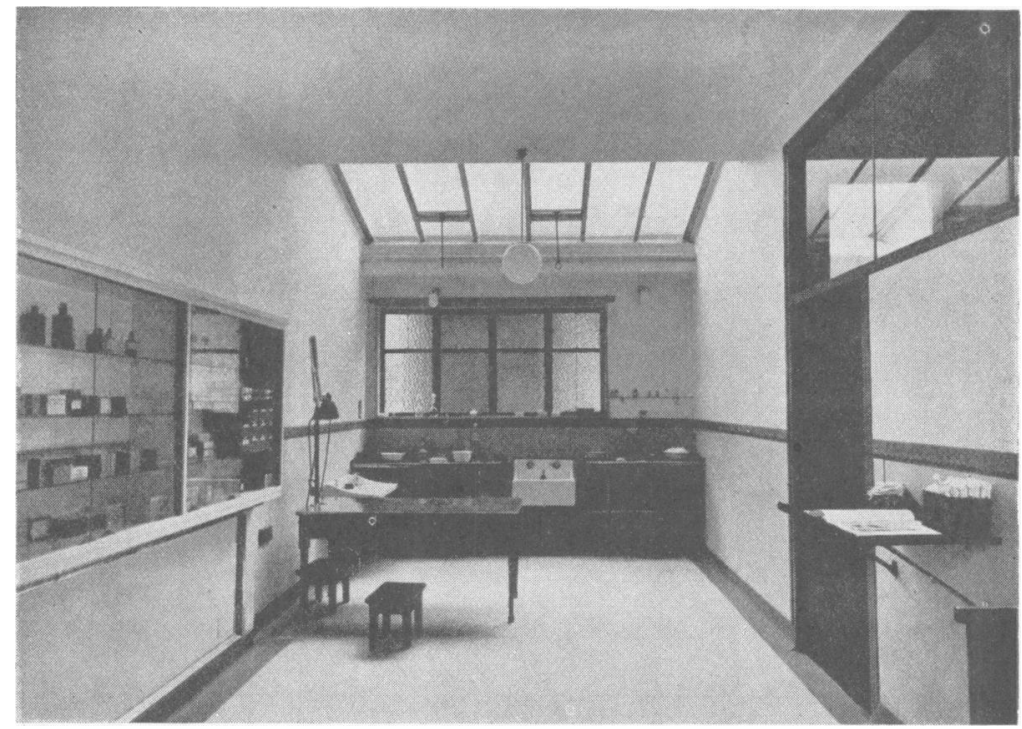

Treatment Room No. 2 (for Treatment of Syphilis).

allocated for male patients, and the remainder has been designed for use as the general out-patient department, as here the best light is available and roof lighting is provided.

Several new features in hospital design are incorporated in this building.

The radiators are of a new panel type and are fitted into the walls below the windows. Thus the walls and floors are clear of projections and can be easily kept clean. A double cove skirting is so placed that beds and furniture cannot damage the walls and paintwork. The floors are laid with thick linoleum of buff and green 


\section{BRITISH JOURNAL OF VENEREAL DISEASES}

colour direct on the concrete, giving an area free from joints, silent, comfortable to walk upon and of pleasing appearance. The doors are all of the flush pattern (one piece) in Gaboon mahogany finished with French polish. All the lighting fittings are of the latest enclosed type, the corridors having panel lighting and a special arrange-

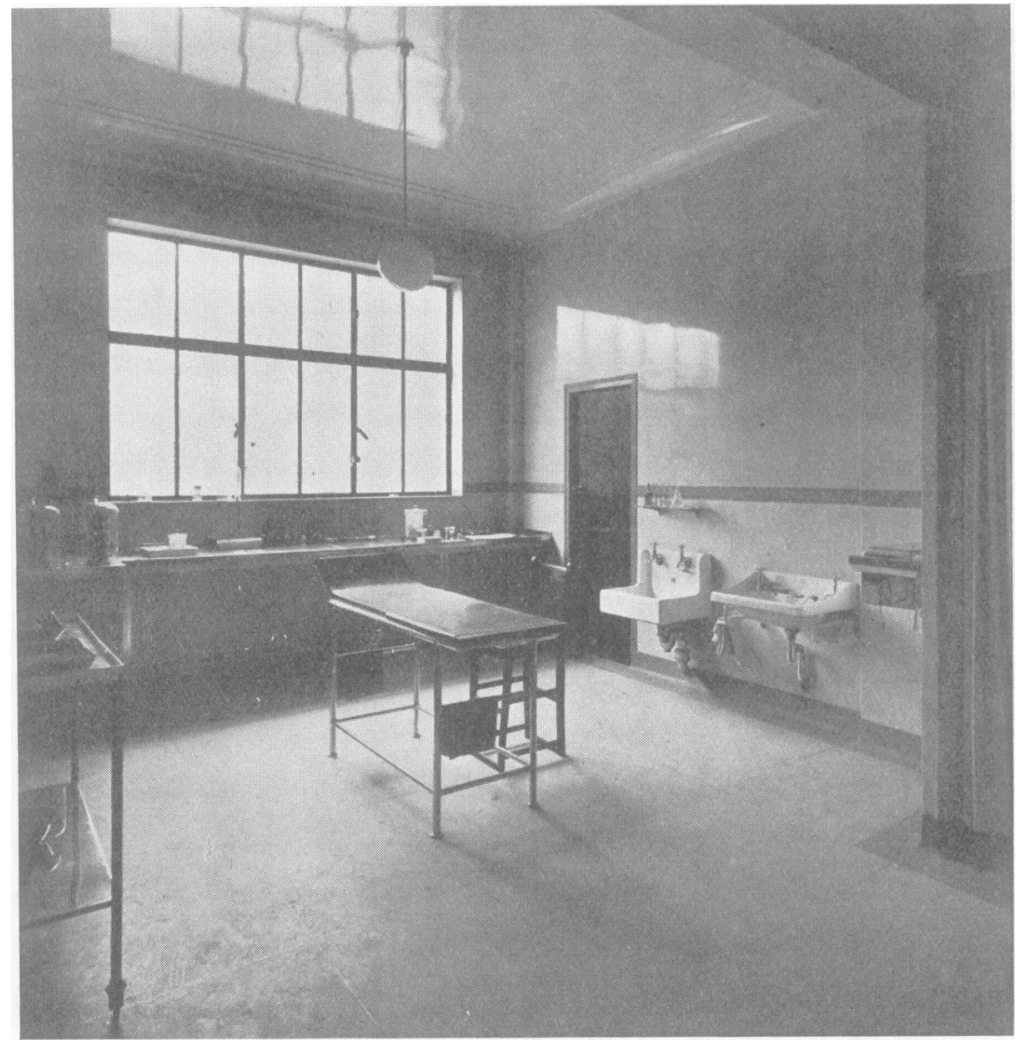

Examination Room No. I.

ment of night lights, 2 feet from the floor, which prevents the rays penetrating the wards at the fanlights and so disturbing the patients. The decoration of the new department is in keeping with that throughout the remainder of the hospital. The colour scheme in general is white ceilings and walls in cream to buff shades, with light green margins at the doors and the windows. All treatment rooms, bathrooms and toilet rooms are finished with marble terrazzo in the same colours. 


\section{THE ROYAL INFIRMARY OF EDINBURGH}

\section{The New Venereal Diseases Department}

The new Venereal Diseases Department remains a part of the Royal Infirmary, and patients in the wards and clinics are patients of the Royal Infirmary, and escape the stigma which inevitably attaches to those attending an ad hoc clinic. The cover thus afforded to patients is of great value in securing regular attendance, the psychological effect being such as to promote the confidence and freedom from worry which are important factors in maintaining the general health of the patient.

The venereal diseases patients enter the infirmary by the west gate and gain access to the department by the main entrance, which is on the south side facing the Eye Pavilion. Female patients are then conveyed to the second floor by a lift, and men proceed to the office which adjoins the large waiting room on the ground floor, as shown in the architect's plan. Male patients will normally enter the building by the north door. At the office the male patient gives his particulars to the nursing orderly in charge, who enters in the register the patient's name, address, age, occupation, and doctor recommending, and gives the patient a card bearing an identification number, by which the patient is distinguished during his period of treatment. The identification card contains information about the times when the patient should present himself for medical inspection and routine treatment.

After receiving his identification number, the new patient is directed to the small waiting room reserved for new arrivals, and those attending subsequently for medical inspection take seats in the large waiting room. The office is equipped with files and index cabinets for the storage of records, and adjoining it is a room for the Secretary, whose services are available for the clerical work of administration.

Prominently displayed high up on the wall of the waiting room is an electrically lighted and operated indicator, by means of which a doctor in any of the five examination rooms can summon a patient. The two largest of the five examination rooms have dressing cubicles and the new patients will be examined in one or other of these larger rooms. The floor space in these rooms is sufficient to accommodate small groups of 


\section{BRITISH JOURNAL OF VENEREAL DISEASES}

students, enabling them to inspect cases at close quarters and watch the work of diagnosis and treatment being carried out. Each of the examination rooms is furnished with a simple type of operating and inspection table, on which the patient lies comfortably on "Dunlopillo" cushions. A wash-hand basin for the doctor, a urine sink and work-bench for microscopical and side-room work are provided in each room.

Doors from the waiting room and also from the main corridor open into the irrigation room, which contains twelve individual irrigation cubicles, six wash-hand basins, two sitz baths, an inspection table curtained off for dressings, shelves for stock lotions, etc. The irrigation cubicles have been arranged to afford privacy and protection for the patient, and at the same time allow the orderly to inspect, to teach the new patient, and satisfy himself that each patient is irrigating properly. Glass screens are fitted in the cubicles in front of the drainage trough to prevent the soiling of the floors with lotions. The sitz baths are valuable adjuncts in the treatment of such complications as prostatitis, seminal vesiculitis and epididymitis.

The improved facilities in the irrigation room will result in a great saving of time for patient and nursing orderly. The patient can wash his hands quickly before and after irrigation, and the orderly has easy access to the stock lotion bottles and dressings. Next to the irrigation room is a small waiting room, which is also used by patients for injection treatment. The injection room has a table with a top of stainless metal for resting the arms for intravenous injection, cupboards for storing drugs, glass-ware, etc., and a basin and sink for washing up. A special supply of sterile water has been arranged, which will greatly facilitate the cleansing of syringes, beakers and basins. Adjoining the injection room is a room for urethroscopic or other special operative treatment. This room contains a more elaborate operating table and cabinets for instruments. A room to accommodate sterilisers and one for the storage of drugs and dressings are provided. After urethroscopy, lumbar puncture, or other minor operative procedures, a patient may be detained under observation in the rest and recovery room. On the ground floor also there are the director's room, from which the administration of the department 


\section{THE ROYAL INFIRMARY OF EDINBURGH}

will be carried on, and the assistant doctor's room, with tables and bookcases for books and periodicals.

The first floor plan includes a large, well-lighted and equipped fourteen-bed ward, two small two-bed wards, and two one-bed wards, with the usual offices, a convalescent and day room, where the up-patients take their meals, and, in addition, a lecture theatre. The large ward has a balcony facing south and catching the sun for the greater part of the day. The small wards ensure greater privacy for special cases-for example, those requiring the frequent application of dressings to the genitals. The second floor accommodates the female wards and the female out-patient department. The clinic rooms occupy the west end of the floor, and are grouped and arranged to facilitate the speedy handling of large numbers of out-patients.

An interesting innovation is the provision of a pedestal closet (Clinette) for the easy collection of specimens of urine. In general design this new appliance is not unlike the W.C. pan, and the expelled urine is directed into a large test glass conveniently placed for its reception. The porcelain construction and water supply render cleanliness easily attainable.

Returning to the ground floor, this houses a complete male out-patient clinic, and the lay-out has been designed with a view to securing improvements on the old department in many respects. In the first place, every effort has been made to provide such facilities as will attract patients to the clinic and secure their regular attendance. The amenities of the new building are many. The marble-terrazzo walls and floors are clean, and the colour scheme pleasing to the eye. The chromium-plated fittings add brightness and increase the impression of cleanliness, which in the old clinic was impaired by dust-gathering pipes around the walls, chipped enamel fittings and rusted metal. The new premises are commodious, well lighted by daylight and artificial light, well heated and well ventilated, and so arranged as to secure the maximum economy in time both for the patient and the staff, and to ensure that the time occupied in the examination and treatment of each patient be reduced to a minimum. 